\title{
FIXED POINT THEOREMS FOR MAPPINGS WITH A CONTRACTIVE ITERATE AT A POINT
}

\author{
L. F. GUSEMAN, JR.
}

Abstract. In a recent paper, V. M. Sehgal discussed continuous self-mappings of a complete metric space with a contractive iterate at each point of the space. Sehgal showed that such mappings have a unique fixed point and the sequence of iterates of any point in the space converges to the fixed point. In the present paper, the result of Sehgal is generalized to mappings which are not necessarily continuous and which have a contractive iterate at each point in a (possibly proper) subset of the space.

1. Introduction. Let $(X, d)$ be a metric space and $f: X \rightarrow X$ a mapping. Let $f^{n}$ denote the $n$th iterate of $f\left(f^{0}(x)=x\right)$, and, for $A \subset X$, let cl $A$ represent the closure of $A$.

In a recent paper, $[1], V . M$. Sehgal proved the following result.

THEOREM 1. Let $(X, d)$ be a complete metric space, and $f: X \rightarrow X$ a continuous mapping satisfying the condition: there exists $a k<1$ such that for each $x \in X$, there is a positive integer $n(x)$ such that for all $y \in X$

$$
d\left(f^{n(x)}(y), f^{n(x)}(x)\right) \leqq k d(y, x) .
$$

Then $f$ has a unique fixed point $u$ and $f^{n}\left(x_{0}\right) \rightarrow$ for each $x_{0} \in X$.

In this paper we investigate mappings which are not necessarily continuous, and satisfy a weaker form of (1) for a (possibly proper) subset of $X$. We present results which extend Theorem 1 and show furthermore that this theorem remains true when the hypothesis of continuity is removed. Examples are given to show that our results are indeed extensions.

The author is grateful to the referee for suggesting the improved version of Theorem 3 presented in this paper.

2. The proof of the following lemma and subsequent theorem follow the line of argument presented in [1].

Lemma. Let $(X, d)$ be a metric space and $f: X \rightarrow X$ a mapping. Let $B \subset X$ with $f(B) \subset B$. If there exists $a u \in B$ and a positive integer $n(u)$ such that $f^{n(u)}(u)=u$ and

Received by the editors January 28, 1970.

AMS 1969 subject classifications. Primary 4785, 5485; Secondary 2654.

Key words and phrases. Fixed points, contraction mapping, contractive iterate. contractive iterate at a point, sequence of iterates. 


$$
d\left(f^{n(u)}(x), f^{n(u)}(u)\right) \leqq k d(x, u)
$$

for some $k<1$ and all $x \in B$, then $u$ is the unique fixed point of $f$ in $B$ and $f^{n}\left(y_{0}\right) \rightarrow u$ for each $y_{0} \in B$.

Proof. By (2), $u$ is the unique fixed point for $f^{n(u)}$ in $B$. Then $f(u)=f\left(f^{n(u)}(u)\right)=f^{n(u)}(f(u))$ implies that $f(u)=u$. But then $u$ is the unique fixed point of $f$ in $B$. Let $y_{0} \in B$, and note that $f(B) \subset B$ implies that $\left\{f^{n}\left(y_{0}\right): n \geqq 1\right\} \subset B$. Let

$$
\alpha\left(y_{0}\right)=\max \left\{d\left(f^{m}\left(y_{0}\right), u\right): 1 \leqq m \leqq n(u)-1\right\}
$$

and for $n$ sufficiently large, write $n=r \cdot n(u)+s$ with $r>0,0 \leqq s<n(u)$. Then

$$
\begin{aligned}
d\left(f^{n}\left(y_{0}\right), u\right) & =d\left(f^{r \cdot n(u)+s}\left(y_{0}\right), f^{n(u)}(u)\right) \\
& \leqq k d\left(f^{(r-1) n(u)+s}\left(y_{0}\right), u\right) \leqq \cdots \\
& \leqq k^{r} d\left(f^{s}\left(y_{0}\right), u\right) \leqq k^{r} \alpha\left(y_{0}\right),
\end{aligned}
$$

and it follows that $f^{n}\left(y_{0}\right) \rightarrow u$.

THEOREM 2. Let $(X, d)$ be a complete metric space and let $f: X \rightarrow X$ be a mapping. Suppose there exists $B \subset X$ such that

(a) $f(B) \subset B$,

(b) for some $k<1$ and each $y \in B$ there is an integer $n(y) \geqq 1$ with $d\left(f^{n(y)}(x), f^{n(y)}(y)\right) \leqq k d(x, y)$ for all $x \in B$, and

(c) for some $x_{0} \in B, \operatorname{cl}\left\{f^{n}\left(x_{0}\right): n \geqq 1\right\} \subset B$.

Then there is a unique $u \in B$ such that $f(u)=u$ and $f^{n}\left(y_{0}\right) \rightarrow u$ for each $y_{0} \in B$. Furthermore, if $d\left(f^{n(u)}(x), f^{n(u)}(u)\right) \leqq k d(x, u)$ for all $x \in X$, then $u$ is unique in $X$ and $f^{n}\left(x_{0}\right) \rightarrow u$ for each $x_{0} \in X$.

Proof. If $y \in B$, one can easily show that $r(y)=\sup _{n} d\left(f^{n}(y), y\right)$ is finite. For the $x_{0} \in B$ hypothesized in (c), let $m_{0}=n\left(x_{0}\right), x_{1}=f^{m_{0}}\left(x_{0}\right)$, and inductively (because of (a) and (b)) let $m_{i}=n\left(x_{i}\right), x_{i+1}=f^{m_{i}}\left(x_{i}\right)$. By routine calculation one can show that the following inequalities hold:

$$
\begin{aligned}
d\left(x_{i+1}, x_{i}\right) & \leqq k^{i} d\left(f^{m_{i}}\left(x_{0}\right), x_{0}\right) \leqq k^{i} r\left(x_{0}\right), & & i \geqq 1, \\
d\left(x_{j}, x_{i}\right) & \leqq \sum_{l=1}^{j-1} d\left(x_{l+1}, x_{l}\right) \leqq \frac{k^{i}}{1-k} r\left(x_{0}\right), & & j>i .
\end{aligned}
$$

It follows that the sequence $\left\{x_{i}\right\}$ is Cauchy. Using completeness and (c) we have $x_{i} \rightarrow u \in B$. Thus there is an integer $n(u) \geqq 1$ such that

$$
d\left(f^{n(u)}(y), f^{n(u)}(u)\right) \leqq k d(y, u)
$$

for each $y \in B$. It follows that $f^{n(u)}\left(x_{i}\right) \rightarrow f^{n(u)}(u)$. Then 


$$
d\left(f^{n(u)}(u), u\right)=\lim _{i} d\left(f^{n(u)}\left(x_{i}\right), x_{i}\right)
$$

However,

$$
\begin{aligned}
d\left(f^{n(u)}\left(x_{i}\right), x_{i}\right) & =d\left(f^{m i-1} \cdot f^{n(u)}\left(x_{i-1}\right), f^{m_{i-1}}\left(x_{i-1}\right)\right) \\
& \leqq k d\left(f^{n(u)}\left(x_{i-1}\right), x_{i-1}\right) \leqq \cdots \\
& \leqq k^{i} d\left(f^{n(u)}\left(x_{0}\right), x_{0}\right) \rightarrow 0 \quad \text { as } i \rightarrow \infty .
\end{aligned}
$$

Hence $f^{n(u)}(u)=u$. By the lemma, $u$ is the unique fixed point of $f$ in $B$ and $f^{n}\left(y_{0}\right) \rightarrow u$ for each $y_{0} \in B$.

The last assertion of the theorem follows directly from the lemma. This completes the proof.

REMARKS. Since $x_{n} \rightarrow u$ implies $f^{n(u)}\left(x_{n}\right) \rightarrow f^{n(u)}(u)$ for any $f$ satisfying (2) in the lemma, the proof of the following theorem follows immediately from the lemma.

TheOREM 3. Let $(X, d)$ be a metric space, $f: X \rightarrow X$ a mapping, and $u, y_{0} \in X$ with $f^{n}\left(y_{0}\right) \rightarrow u$. If $f$ satisfies (2) for all $x \in X$, then $f$ has $a$ unique fixed point $u \in X$ and $f^{n}\left(x_{0}\right) \rightarrow u$ for each $x_{0} \in X$.

3. In this section we present three examples which show that Theorem 2 is an extension of Theorem 1 (even when the continuity condition is removed from the latter). All sets have the usual metric.

ExAmple 1. Define $f:[0,1] \rightarrow[0,1]$ by

$$
\begin{aligned}
f(x) & =\frac{1}{2}, & & \text { if } x \text { irrational, } \\
& =1-x, & & \text { if } x \text { rational. }
\end{aligned}
$$

Then $f\left(\frac{1}{2}\right)=\frac{1}{2}$. For any $k<1$, let $B=\left\{\frac{1}{2}\right\} \cup A$, where $A$ is any collection of irrationals in $[0,1]$. Simple computations show that $B$ cannot contain any rational $x \neq \frac{1}{2}$.

Example 2. Define $f:[0,1] \rightarrow[0,1]$ by

$$
\begin{aligned}
f(x) & =1-x, & & \text { if } x \text { irrational, } \\
& =x, & & \text { if } x \text { rational. }
\end{aligned}
$$

Then each rational $x$ is a fixed point. For any $k<1$, let $B=\{x\}, x$ rational.

EXAMPLE 3. Let $B=\{0\} \cup$ irrationals in $[0,1)$ and define $f:[0,1)$ $\rightarrow[0,1)$ by

$$
\begin{aligned}
f(x) & =0, & & x \in B, \\
& =2^{-n} x, & & x \in\left[2^{-n}, 2^{-(n-1)}\right) \sim B, \quad n=1,2, \cdots .
\end{aligned}
$$


Then 0 is the unique fixed point for $f$ in $[0,1)$. For each $x_{0} \in[0,1)$, $f^{n}\left(x_{0}\right) \rightarrow 0$ and

$$
d\left(f\left(x_{0}\right), f(0)\right) \leqq \frac{1}{2} d\left(x_{0}, 0\right) .
$$

We shall show that there exists $x_{0} \in[0,1)$ such that for each $m \geqq 1$ there exists $x_{m} \in[0,1)$ with

$$
d\left(f^{m}\left(x_{m}\right), f^{m}\left(x_{0}\right)\right)=d\left(x_{m}, x_{0}\right) .
$$

Let $x_{0}=\frac{1}{2}$ and let $\alpha(m)=2^{m}, m \geqq 1$. The following properties of $f$ may be easily verified:

(i) $f^{m}\left(\frac{1}{2}\right)=2^{-\alpha(m)}$.

(ii) $f^{m+1}\left(\frac{1}{2}\right)=\left[f^{m}\left(\frac{1}{2}\right)\right]^{2}=\left[f\left(\frac{1}{2}\right)\right]^{\alpha(m)}$.

(iii) $f^{m}(x)=4 \cdot\left[f^{m}\left(\frac{1}{2}\right)\right]^{2} x$ if $2^{-2} \leqq x<2^{-1}$.

For each $m \geqq 1$, let $y_{m}=\left(2^{\alpha(m)}+2\right)^{-1}$. By using (i)-(iii) above we have

$$
d\left(f^{m}\left(\frac{1}{2}\right), f^{m}\left(\frac{1}{2}-y_{m}\right)\right)=d\left(\frac{1}{2}, \frac{1}{2}-y_{m}\right), \quad m \geqq 1 .
$$

Thus $f$ has no contractive iterate at $\frac{1}{2}$.

\section{REFERENCES}

1. V. M. Sehgal, $A$ fixed point theorem for mappings with a contractive iterate, Proc. Amer. Math. Soc. 23 (1969), 631-634.

Texas A \& M University, College Station, Texas 77843 